\title{
Téoros
}

Revue de recherche en tourisme

\section{Les périodiques étrangers : des outils de travail accessibles}

\section{Jean Pelletier, Martine Racine et Sébastien Servant}

Volume 1, numéro 2, 2e trimestre 1982

La recherche en tourisme : forces et faiblesses

URI : https://id.erudit.org/iderudit/1080857ar

DOI : https://doi.org/10.7202/1080857ar

Aller au sommaire du numéro

Éditeur(s)

Université du Québec à Montréal

ISSN

0712-8657 (imprimé)

1923-2705 (numérique)

Découvrir la revue

Citer ce document

Pelletier, J., Racine, M. \& Servant, S. (1982). Les périodiques étrangers : des outils de travail accessibles. Téoros, 1(2), 24-27.

https://doi.org/10.7202/1080857ar d'utilisation que vous pouvez consulter en ligne. 


\title{
Les périodiques étrangers: des outils de travail accessibles
}

\author{
Par Jean Pelletier, Martine Racine et Sébastien Servant
}

Même si les intervenants et les institutions québécoises n'ont pas su jusqu'ici donner un véritable élan à la recherche touristique dans le but de reculer les limites des connaissances scientifiques et techniques, il faut savoir qu'un grand nombre de chercheurs à travers le monde ont publié des tas de rapports, études et monographies. Le Centre d'études du tourisme (CET) possède près de 12,000 documents de cette nature.

II faut également savoir que la plupart des chercheurs ont publié des articles dans plusieurs revues. Ces périodiques sont des instruments fort utiles pour ceux et celles qui s'intéressent au développement du tourisme et qui cherchent à asseoir leurs réflexions et leurs actions sur les données et les analyses disponibles.

Au delà des grandes recherches qui ont un certain caractère spéculatif, la plupart des publications périodiques essaient de faire connaître des textes orientés sur le changement et susceptibles d'effets pratiques prévisibles. Le présent article veut présenter quelques-unes de ces revues disponibles au CET.

\section{Annals of Tourism Research}

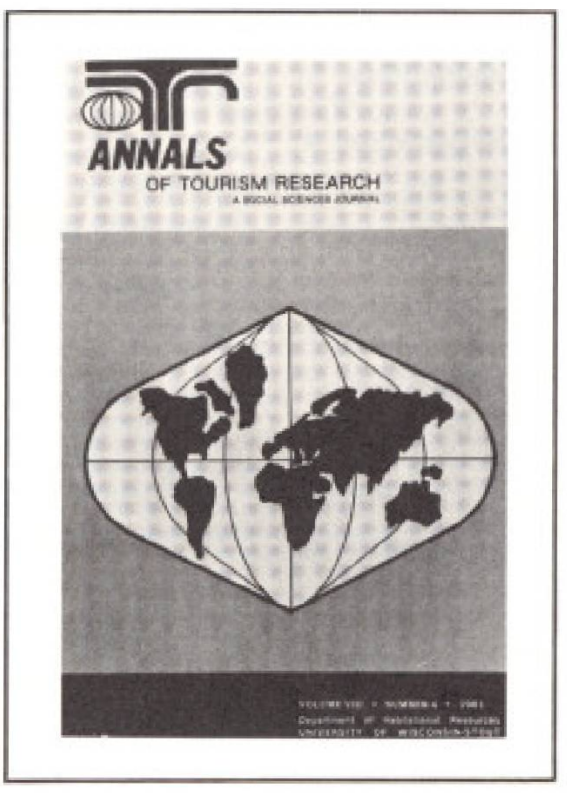

Annals of Tourism Research est une revue de sciences sociales centrée sur le cóté académique du tourisme. Le but de Annals est d'explorer le domaine du tourisme en tant que discipline et matière socio-économiques, d'encouger le développement et la diffusion des recherches sur le tourisme et de faciliter l'application des données et des cadres théoriques aux problèmes académiques aussi bien que pratiques du phénomène touristique. Quoique Annals considère le tourisme comme domaine particulier, la revue estime quion ne réussira à élargir les connaissances du tourisme que par un effort pluridisicplinaire. Annals sert de forum où peuvent se rencontrer les diverses disciplines engagées sur le tourisme.
Le fondateur et responsable de cette publication, de ce trimestriel qui entre dans sa neuvième année d'existence, Jafar Jafari, travaille à l'Université de Wisconsin-Stout (Department of Habitational Resources). C'est cette dernière qui a assuré jusqu'à récemment la publication de Annals. Depuis janvier 1982, c'est une des grandes maisons d'édition. Pergamon Press, qui assure la production et la diffusion de la revue.

Le numéro d'automne 1981 contient trois grands articles:

a) Dimensions of the social group role in pleasure vacations:

b) Touring by automobile in 1932: the american West as Stereotype.

c) A segmentation analysis of resident attitudes to the social impact of tourism (cet article est co-signé par J.E. Brougham de I'Université Laval et par R.W. Butler (Western Ontario).

Notons que les articles font l'objet d'un résumé en français. En plus des grands articles, chaque numéro présente aussi des comptes rendus de livres, des notes de publication et des rapports de congrès.

Comme a l'habitude, un index cumulatif de Annals paraît dans le dernier numéro de chaque volume annuel. Ainsi celui de 1981 comprend un index chronologique et un index d'auteurs pour les huit volumes précédents. 


\section{Bulletin d'études touristiques}

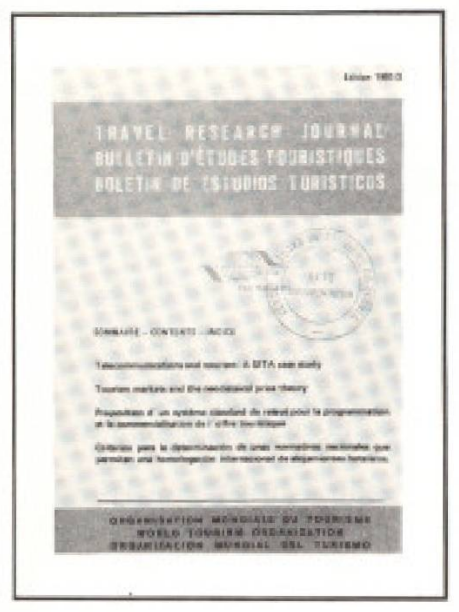

"L'objectif fondamental de I'Organisation mondiale du tourisme est de promouvoir et de développer le tourisme en vue de contribuer à l'expansion économique, à la compréhension internationale, à la paix, à la prospérité ainsi qu’au respect universel et à l'observation des droits et des libertés humaines fondamentales sans distinction de race, de sexe, de langue ou de religion". (Statuts de I'OMT). Le CET est membre de I'OMT.

Une des tâches essentielles de I'OMT est de fournir à ses membres une connaissance permanente du phénomène touristique et de ses incidences sur la vie sociale, économique et culturelle des nations. $\mathrm{A}$ cette fin, le Bulletin d'études touristiques vise essentiellement à présenter des théories, des méthodologies et des concepts susceptibles de susciter des innovations pratiques en matière de recherche touristique et de faciliter l'étude et l'analyse objective de ce domaine de l'activité humaine.

Pour atteindre ces objectifs, le Bulletin d'études touristiques et ses responsables, R.C. Lonati et R. Lanquar sont ouverts à la collaboration des chercheurs, experts, professionnels et responsables du tourisme à qui il est ainsi offert un moyen d'expression et de diffusion des idées, sur des sujets ou des thèmes proches des préoccupations de I'Organisation dans tous les domaines et les disciplines d'étude du tourisme. Ce bulletin annuel diffuse ses articles en 3 langues (français, anglais, espagnol).

L'édition 1981/82 contient par exemple une proposition du professeur Peroni (Rome) d'un système standard de relevé pour la programmation et la commercialisation de l"offre touristique. II s'agit là d'un instrument technique pour photographier un "état de fait": ce relevé pourrait constituer la base fondamentale d'une documentation qui est indispensable aussi bien aux techniciens s'attachant à la disposition préalable des plans d'aménagement qu'aux organismes de promotion du tourisme. Le professeur Peroni propose les fiches-standards suivantes: fiches de la capacité du développement touristique de chaque localité, de relevé des caractéristiques structurelles et touristiques des terrains de camping et de caravaning. de relevé pour les résidences et villages touristiques à vocation non-hốtelière et des relevés pour bungalows et logements prévus en location touristique.

\section{BITS information}

Le Bureau international du tourisme social (BITS) est une association fondée en 1963 qui regroupe maintenant 92 organisations nationales relevant de 25 pays (dont le Centre d'études du tourisme, le Regroupement des organismes nationaux de loisir du Québec et la Société VacancesFamilles). 11 organismes internationaux, 12 organisations gouvernementales (dont I"Office du Tourisme du Canada et le ministère du Loisir, de la Chasse et de la Pêche du Québec).

Son principal objectif est de promouvoir le développement du tourisme social dans le monde en favorisant les échanges d'informations et d'expériences entre ses membres. Pour ce faire, le BITS organise des congrès et des séminaires internationa ux consacrés à l'examen de l'évolution du tourisme social. II veut s'affirmer comme un carrefour d'idées, un point de conjugaison des efforts internationaux de promotion du tourisme social.

BITS Information est un périodique trimestriel qui cherche à donner une information universelle sur tous les aspects, problemes et ambitions du tourisme social international. La plupart des textes sont publiés en français: I'éditorial et les informations diverses sont reproduits dans cinq langues. Depuis quelques numéros, la revue contient un supplément sur le tourisme coopératif.

Le numéro 68 (mars 82) présente des informations sur le tourisme rural en Turquie, en Wallonie et en France et un article sur l'avenir du tourisme des jeunes.
Espaces

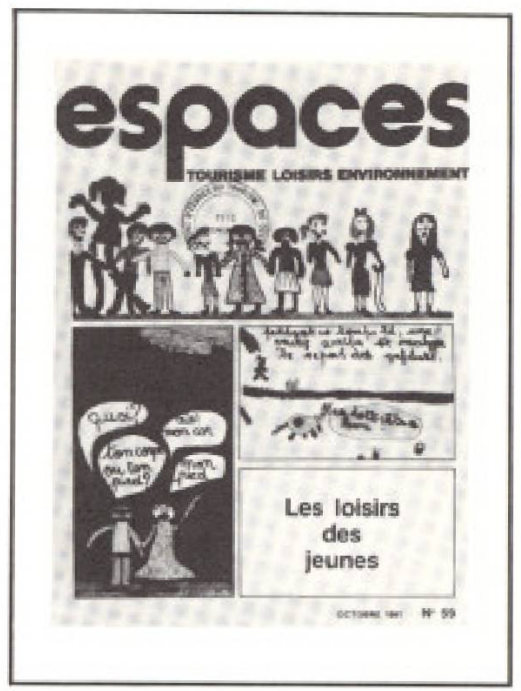

Espaces est une revue française sous titree depuis ses débuts Tourisme Loisirs Environnement. D'abord trimestrielle, Espaces est devenue mensuelle en 1979. Elle a cependant suspendu sa parution en novembre dernier et doit reprendre en juin 1982.

Espaces a toujours voulu jouer un rôle de carrefour et de trait d'union. Son équipe formée de spécialistes, de professeurs et de techniciens est soucieuse de livrer une réflexion sur I'actualité touristique de plus en plus complexe et de plus en plus intégrée dans le tissu économique et sociologique.

La revue s'adresse tant aux décideurs des secteurs public et privé qu'aux chercheurs et enseignants. Elle se définit comme la revue de tous ceux qui concourent à aménager le temps et I'espace pour I'agrément et la qualité de vie de tous.

Depuis deux ans, Espaces est dirigé par Yves Raynouard. Tout en faisant une large part aux informations pratiques et aux dossiers d'actualité intéressants tant les régions que les professions touristiques, la revue publie des articles et études plus fouillés.

Au sommaire des numéros de l'année 1981, signalons des dossiers sur la nouvelle politique française du temps libre, les régions d'accueil (tourisme et aménagement rural), le tourisme fluvial, les exigences d'une économie des loisirs et les parcs naturels.

Dans le No 59 (octobre 1981), on retrouve en plus d'un dossier sur les loisirs des jeunes en vacance, des textes sur la naissance du tourisme social au Mexique, la formation, la recherche et des informations d'actualité. 


\section{Leisure, Recreation and Tourism Abstracts}

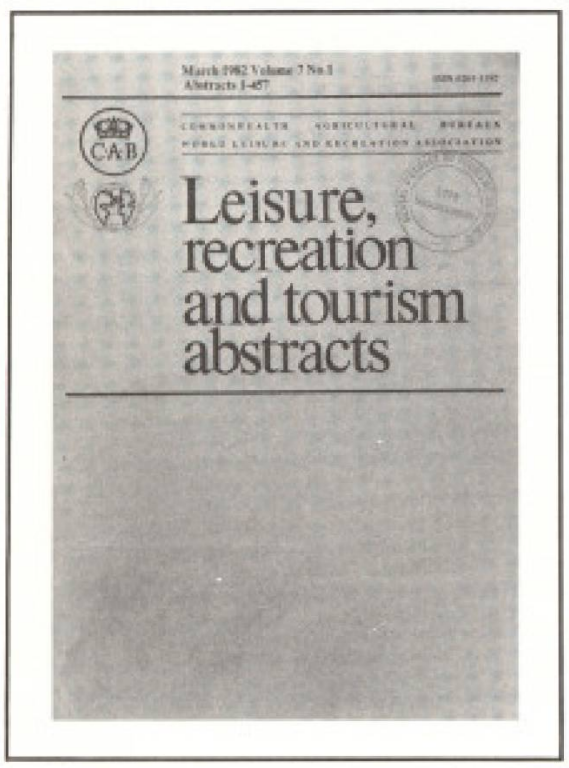

A l'origine (1976) jusqu'au début de 1981, la publication portait le titre de Rural Recreation and Tourism Abstracts.

Leisure, Recreation and Tourism Abstracts est une publication trimestrielle maintenant parrainée conjointement par les Commonwealth Agricultural Bureaux (CAB) et la World Leisure and Recreation Association. Elle est préparée par le Commonwealth Bureau of Agricultural Economics et publié par les $\mathrm{CAB}$.

Cette publication illustre l'élargisse. ment du concept de ruralité et témoigne de la volonté de ne pas couper le développement du milieu rural des économies nationales. Les $\mathrm{CAB}$ produisent 45 autres publications pour couvrir l'ensemble des thèmes reliés à l'agriculture.

De fait, Leisure Recreation and Tourism Abstracts constitue un index et une liste bibliographique annotée de tout ce qui se publie à travers le monde. Le dernier numéro (mars 1982) présente 457 documents larticles ou livres). En 1981, 1,671 résumés avaient été ainsi présentés.

Les notes bibliographiques sont classées par catégories: culture and entertainment, home and neighbourhood activities, natural resources, recreation activities and facilities, tourism et ce en plus d'une première catégorie générale (theory, policy and planning, etc) qui porte le même titre que la publication.

\section{Politique du tourisme et tourisme international dans les pays membres de I'OCDE}

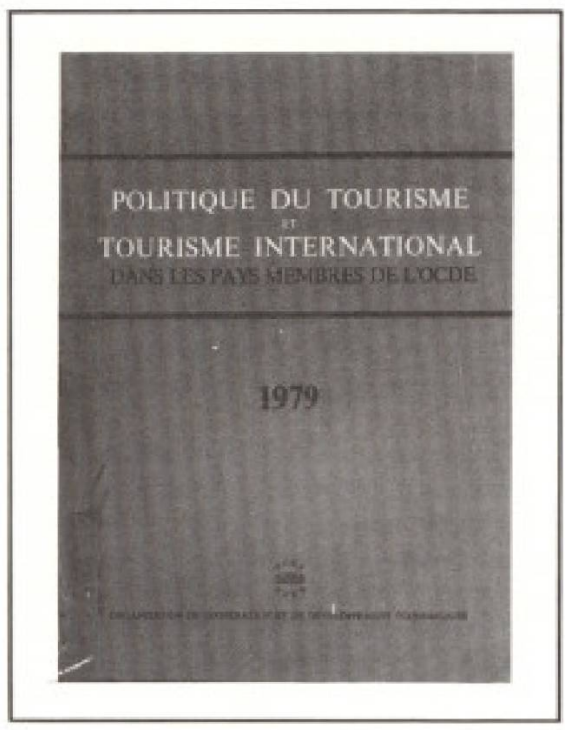

L'Organisation de coopération et de développement économique a été constituée en 1960 par 18 pays européens ainsi que par le Canada et les Etats-Unis. Depuis I'Australie, le Japon et la Nouvelle-Zélande ont joint les rangs de I'OCDE.
L'OCDE a pour objectif de promouvoir des politiques visant à réaliser la plus forte expansion possible de l'économie et de l'emploi et une progression du niveau de vie dans les pays membres et contribuer ainsi au développement du commerce mondial sur une base multilatérale et non discriminatoire.

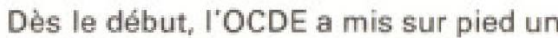
Comité du tourisme - organe réunissant les hauts fonctionnaires et les personnalités responsables. Ce Comité publie un rapport annuel qui contient des vues sur les divers problèmes qui se posent, aussi bien aux gouvernements qu'à certains secteurs économiques, du fait du développement considérable du mouvement touristique au cours des dernières années.

En plus de nombreuses données statistiques, les cinq chapitres habituels portent sur a) la politique et les actions gouvernementales; b) les flux touristiques, c) l'importance économique du tourisme, d) le transport aérien et e) l'hébergement touristique.

La constance des méthodes employées par la plupart des pays membres dans le relevé des statistiques touristiques permet d'établir de façon satisfaisante l'évolution du mouvement touristique dans un même pays d'une année à l'autre.

Par contre, les comparaisons entre les données correspondantes, relatives aux divers pays membres, doivent être effectuées avec précaution car ces données ne répondent pas toujours exactement aux définitions standard et sont recueillies par des méthodes souvent différentes. 
Journal of Travel Research

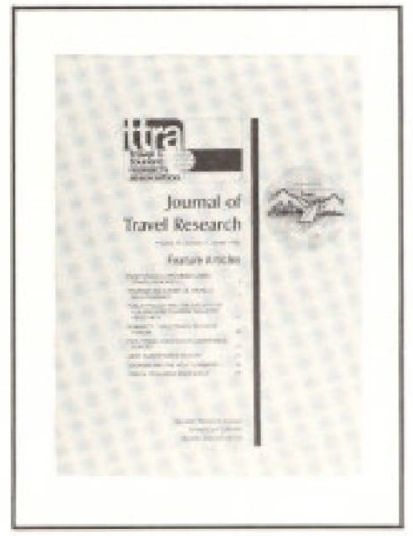

La Travel and Tourism Research Association et les organismes qui I'ont précédé ont contribué depuis une vingtaine d'années au développement du tourisme par l'organisation de séminaires et par la production de plusieurs publications. Le TTRA est un organisme à caractère international dont le membership est essentiellement composé d'organismes et associations américains et canadiens (dont le CET). Le TTRA est actuellement présidé par J.R. Brent Ritchie, anciennement de I'Université Laval et maintenant de l'Université de Calgary.

Le TTRA publie régulièrement une "News Letter" et une "Tourism educators newsletter". Le Journal of Travel Research est la principale publication du TTRA: elle est publiée quatre fois I'an. Parmi les récents articles, signalons à titre d'exemples: Where to find travel research facts /C.R. Goeldner): Travel market segmentation and the implementation of market strategies (B.E. Bryant et A.J. Morrison): Family vacation decisionmaking-a replication and extension (J.R.B. Ritchie et P. Filiatrault): A comprehensive approach to the planning of tourism industry (J. Bargur et A. Arbel).

Dans le dernier numéro (hiver 1982), on retrouve trois articles intéressants à plus d'un titre: a) Positioning a province (Nova Scotia) using travel Research, b) Tourism and crime: is there a relationship? c) Public policy and the growth of the mexican tourism industry. 1970-1979.

Aux articles s'ajoutent quelques notes plus bréves sur des études en cours et des recherches à caractère novateur et une bibliographie annotée de publications récentes (148 dans ce numéro)

Un index de tous les articles publiés depuis 1968 peut être consulté.
Revue

\section{de l'Académie internationale du tourisme}

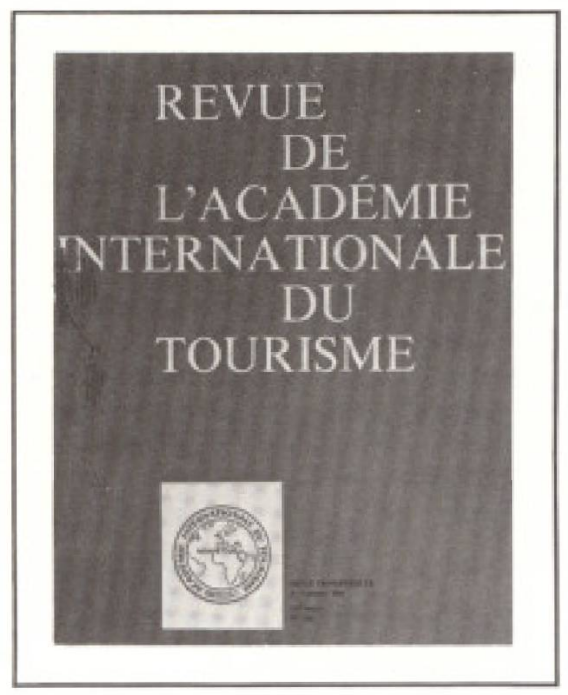

L'article 4 des statuts de l'Académie prévoit qu'elle "a pour mission de développer le caractère culturel et humaniste du tourisme international et d'en codifier le vocabulaire"

L'Académie qui compte une quarantaine de membres existe depuis 1948. Son secrétariat permanent est situé à Monte Carlo. La XXXle assemblée générale (automne 1981) avait pour thème: "quelle influence. heureuse ou néfaste, peut avoir le tourisme en provenance des pays développés sur les traditions, les valeurs spirituelles, la personnalité du pays d'accueil, particulièrement si celui-ci est un pays en voie de développement?"

La revue de l'Académie est trimestrielle et la grande majorité des articles sont présentés en français. Ce sont maintenant les Editions Nagel qui assume les frais de publication.

Au sommaire des derniers numeros, on peut trouver des textes comme:

- L'humanisme contemporain et les voyages;

- European tourism: future prospects 1980-1990;

- Les techniques de gestion touristique moderne au service d'un tourisme plus humain.

Chaque numéro comporte également une chronique "échos du tourisme".

Signalons enfin que l'Académie a édité dès ses débuts un dictionnaire touristique international. II est continuellement remis à jour et maintenant publié en douze langues.
Revue du Tourisme

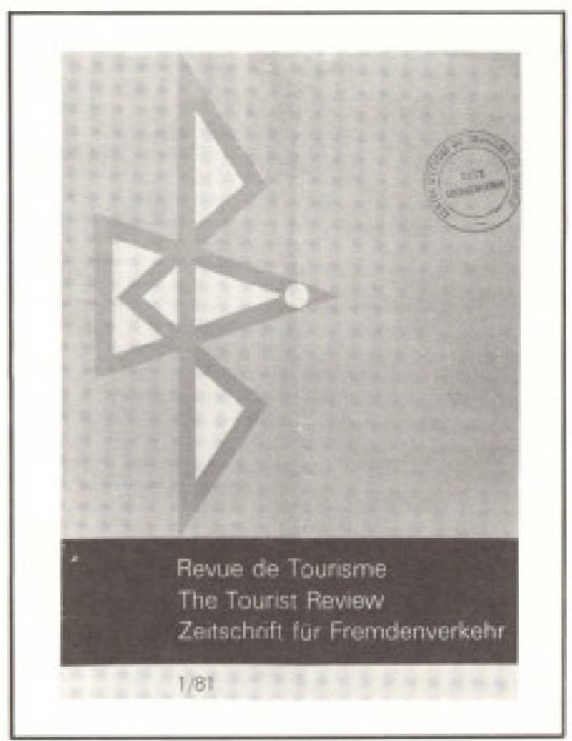

La Revue du Tourisme, qui parait quatre fois l'année, est présentée comme l'organe officiel de I'Association internationale d'experts scientifiques du tourisme (AIEST). Les textes de la revue sont publiés en allemand, en anglais ou en francais.

Ce périodique est publié depuis 1945 par les mêmes éditeurs: I'Institut de recherches touristiques de I'Université de Berne et I'Institut de tourisme et d'économie des transports à l'École des Hautes études économiques et sociale de Saint-Gall.

La publication qui cherche à "garder son caractère d'éditorial du monde scientifique du tourisme" a cependant ajouté au cours des récentes années quelques nouvelles rubriques pour suivre l'actualité: revue des livres, bibliographie et chronique sur les institutions proches de l'AIEST.

Voici, à titre d'exemples, certains textes publiés depuis le début de 1980 .

- Essai systématique d’un inventaire des ressources touristiques;

- L'offre de produits touristiques en économie de marché: concurrence ou monopole?;

- Quelques réflexions sur la notion de "capacité de charge" dans le processus du développement du tourisme:

- Limites de l'aménagement touristique de l'espace rural.

Signalons également la contribution de professeurs canadiens comme B. Keogh de Moncton (Motivations and the choice decisions of skiers in No 1/80) et G. Kibedi de Toronto (Future trends in International Tourism in No 1/81). 